\title{
Reversible Semi-fragile Image Authentication Using Zernike Moments and Integer Wavelet Transform
}

\author{
Xiaoyun $\mathrm{Wu}^{1,2}$, Xiaoping Liang ${ }^{1,2}$, Hongmei Liu ${ }^{1,2}$, Jiwu Huang ${ }^{1,2}$, \\ and Guoping Qiu ${ }^{3}$ \\ ${ }^{1}$ School of Information Science and Technology, Sun Yat-Sen University, Guangzhou, \\ Guangdong, 510275, China \\ isshjw@mail.sysu.edu.cn \\ ${ }^{2}$ Guangdong Province Key Laboratory of Information Security, 510275, P.R. China \\ ${ }^{3}$ School of Computer Science, University of Nottingham, NG8 1BB, UK
}

\begin{abstract}
Semi-fragile image authentication based on watermarking has drawn extensive attention. However, conventional watermarking techniques introduce irreversible distortions to the host signals and thus may not be allowable in some applications such as medical and military imaging. Though some reversible fragile authentication algorithms had been developed, reversible semi-fragile authentication presents a challenge. To the best of our knowledge, so far there are only two reversible semi-fragile authentication algorithms based on watermarking reported in the literature. The existing reversible semi-fragile authentication schemes have two shortcomings: i) Watermark security has not received attention; ii) They have weak capability to resist JPEG compression. In this paper, we propose a novel reversible semi-fragile image authentication scheme. The algorithm can distinguish malicious modification from incidental modification according to semi-fragile characteristics of Zernike moments magnitudes (ZMMs) of the low frequency subband in integer wavelet transform (IWT) domain of an image. Combining semi-fragile characteristics of ZMMs, the watermark can discern forgery attack, thus, improving watermark security. The algorithm can locate the tampered area of an image accurately while tolerating JPEG lossy compression at a low quality factor. Experimental results demonstrate the merits of the proposed algorithm.
\end{abstract}

\section{Introduction}

Using powerful image processing software, digital image may be arbitrarily modified. In many cases, a modified image may leave no visual clue of it being tampered with, therefore, in this digital age, "seeing is believing" is not always true anymore. As a consequence, in many real world applications, authentication for the integrity and authenticity of an image becomes an important issue.

A possible authentication technique to solve this problem is to embed a fragile watermark [1-3] or a semi-fragile watermark [4-7] into the image. The fragile watermark is sensitive to any modification imposed on the image which may be applied to exact authentication. However, in many real world applications, digital 
image will inevitably be subjected to content-preserving operations such as JPEG lossy compression, noise, geometrical manipulations (scaling and rotation) etc. In this case, a practically useful authentication system should accept such manipulations even if the image has been modified. Furthermore, the authentication system should be able to detect content-altering manipulations such as cut and replacement. Semifragile watermark is able to fulfill this purpose and is applicable to semi-fragile authentication. Therefore, semi-fragile authentication is more practical than exact authentication. Because most proposed semi-fragile authentication schemes reject geometrical manipulations [8], we only focus the evaluation of semi-fragility on JPEG lossy compression in this paper.

The marking techniques mentioned above may introduce irreversible distortions due to quantization error, truncation error or bit replacement operations in watermarking embedding. In some applications, such as medical or military imaging, these distortions are not acceptable once the image is deemed to be authentic. In other words, once it is authenticated, the original image must be retrieved from the marked image in a lossless manner. The reversible watermarking technique can achieve such a goal.

The existing reversible data hiding algorithms can be classified into three categories [9]: (i) Those developed for high capacity data hiding [10-13]; (ii) Those developed for fragile authentication [14-16]; (iii) Those developed for semi-fragile authentication [17-18]. The main difference between category (i) and category (ii) as well as category (iii) is in their data hiding capacity which category (i) is much higher than that of categories (ii) and (iii) methods. Reversible semi-fragile authentication may have many applications, however, this is an area which has not been well studied. To the best of our knowledge, there are only two reversible semi-fragile authentication algorithms reported in the literature [17-18]. De Vleeschouwer et al. [17] proposed a reversible semi-fragile data hiding scheme based on patchwork theory. It can tolerate JPEG lossy compression at a high quality factor. However, the watermarked images may suffer from salt-and-pepper noise due to module 256 addition. Zou et al. [18] proposed a lossless semi-fragile authentication scheme based on integer wavelet transform (IWT). It adopted 5/3 IWT family recommended by JPEG2000 standard and is robust against compression by JPEG2000. Overflow/underflow and salt-and-pepper noise are avoided by adjusting $L_{L}$ subband coefficients properly. Although the method in [17] is robust against JPEG lossy compression, it can only tolerate compression at a high quality factor. Both [17] and [18] have not tackled watermark security issues. By analyzing potential security vulnerabilities existing in the reversible watermarking schemes, Katzenbeisser et al. [19] generalizes three classes of attacks: the attacks against the key distribution, the attacks in the verification stage and the attacks in the reconstruction stage. Here, we concentrate security concern on the attacks in the verification stage, specifically forgery attack which is a deadly attack for image authentication using watermarking scheme. Such attack can modify image content without altering the mark data. As a result, an image that has been tampered with by forgery attack is able to pass authentication processes. As a good authentication 
algorithm for image using watermarking technique, it should resist forgery attack and tolerate JPEG compression to a certain extent.

In this paper, we propose a novel reversible semi-fragile image authentication scheme. Our new method is able to counter forgery attacks and is also robust against low quality factor JPEG lossy compression. If the marked image has not been modified, our method can retrieve the original image from the marked image. In our method, semi-fragile characteristics of the Zernike moments magnitudes (ZMMs) of the low frequency subband in the IWT domain of an image is used to distinguish malicious attack from incidental attack. By combining semi-fragile characteristics of ZMMs, the watermark can discern forgery attack, thus improving watermark security. The proposed method can locate the tampered region of an image accurately.

The paper is organized as follows. In Section 2, we give the integer wavelet transform of $9 / 7$ biorthogonal wavelet and show the semi-fragile characteristics of the ZMMs of the low frequency subband in the IWT domain of an image. Section 3 describes the proposed algorithm, including outline of the scheme, reversible watermark embedding, tamper detection and recovery. Experimental results and conclusions are given in Section 4 and Section 5, respectively.

\section{Integer Wavelet Transform and Zernike Moments}

\subsection{Integer Wavelet Transform}

Because it can reconstruct the original image without distortion, we adopt IWT to implement reversible watermarking. Specifically, we use lifting scheme to realize IWT of CDF $9 / 7$ biorthogonal wavelet. An example of the lifting of CDF 9/7 biorthogonal wavelet is given in [20]. To one dimensional signal $\left\{x_{l}\right\}_{l \in Z}$, the lifting steps are described as follows.

$$
\begin{aligned}
& \left\{\begin{array} { l } 
{ s _ { l } ^ { ( 0 ) } = x _ { 2 l } } \\
{ d _ { l } ^ { ( 0 ) } = x _ { 2 l + 1 } }
\end{array} \quad \left\{\begin{array} { l } 
{ d _ { l } ^ { ( 1 ) } = d _ { l } ^ { ( 0 ) } + \alpha ( s _ { l } ^ { ( 0 ) } + s _ { l + 1 } ^ { ( 0 ) } ) } \\
{ s _ { l } ^ { ( 1 ) } = s _ { l } ^ { ( 0 ) } + \beta ( d _ { l } ^ { ( 1 ) } + d _ { l - 1 } ^ { ( 0 ) } ) }
\end{array} \left\{\begin{array}{l}
d_{l}^{(2)}=d_{l}^{(1)}+\gamma\left(s_{l}^{(1)}+s_{l+1}^{(1)}\right) \\
s_{l}^{(2)}=s_{l}^{(1)}+\delta\left(d_{l}^{(2)}+d_{l-1}^{(2)}\right)
\end{array}\right.\right.\right. \\
& \left\{\begin{array}{l}
s_{l}=\zeta s_{l}^{(2)} \\
d_{l}=d_{l}^{(2)} / \zeta
\end{array}\right. \\
& \alpha=-1.586134342 ; \beta=-0.05298011854 ; \gamma=0.8829110762 \text {; } \\
& \delta=0.4435068522 ; \zeta=1.149604398
\end{aligned}
$$

where $s_{l}$ and $d_{l}$ are generally referred to as lower frequency and detail coefficients, respectively. $s_{l}^{(i)}, d_{l}^{(i)}(i=0,1,2)$ are mid-outputs.

According to integer wavelet theory [21], we construct integer wavelet transform based on the framework mentioned above. That is: 


$$
\begin{gathered}
\left\{\begin{array}{l}
s_{l}^{(0)}=x_{2 l} \\
d_{l}^{(0)}=x_{2 l+1}
\end{array}\left\{\begin{array}{l}
d_{l}^{(1)}=d_{l}^{(0)}+\operatorname{Int}\left(\alpha\left(s_{l}^{(0)}+s_{l+1}^{(0)}\right)\right) \\
s_{l}^{(1)}=s_{l}^{(0)}+\operatorname{Int}\left(\beta\left(d_{l}^{(1)}+d_{l-1}^{(0)}\right)\right)
\end{array}\right) \begin{array}{l}
d_{l}^{(2)}=d_{l}^{(1)}+\operatorname{Int}\left(\gamma\left(s_{l}^{(1)}+s_{l+1}^{(1)}\right)\right) \\
s_{l}^{(2)}=s_{l}^{(1)}+\operatorname{Int}\left(\delta\left(d_{l}^{(2)}+d_{l-1}^{(2)}\right)\right)
\end{array}\right. \\
\left\{\begin{array} { l } 
{ d _ { l } ^ { ( 3 ) } = d _ { l } ^ { ( 2 ) } + \operatorname { I n t } ( ( \zeta - \zeta ^ { 2 } ) s _ { l } ^ { ( 2 ) } ) } \\
{ s _ { l } ^ { ( 3 ) } = s _ { l } ^ { ( 2 ) } + \operatorname { I n t } ( ( - 1 / \zeta ) d _ { l } ^ { ( 3 ) } ) }
\end{array} \left\{\begin{array} { l } 
{ d _ { l } ^ { ( 4 ) } = d _ { l } ^ { ( 3 ) } + \operatorname { I n t } ( ( \zeta - 1 ) s _ { l } ^ { ( 3 ) } ) } \\
{ s _ { l } ^ { ( 4 ) } = s _ { l } ^ { ( 3 ) } + d _ { l } ^ { ( 4 ) } }
\end{array} \left\{\begin{array}{l}
s_{l}=s_{l}^{(4)} \\
d_{l}=d_{l}^{(4)}
\end{array}\right.\right.\right.
\end{gathered}
$$

where $\operatorname{Int}(x)$ means integer part of $x$. The values of parameters $\alpha, \beta, \gamma, \delta, \zeta$ are given in formula (3). Equation (5) is an extra lifting step different from Equation (2). We adopt it here because it can achieve reversible transform according to [19] while Equation (2) cannot.

\subsection{Zernike Moments}

Zernike moments of a digital image are widely applied to pattern recognition, target classification, target identification and scene analysis. For a definition of the Zernike moments of order $n$ with repetition $m$ of a digital image, please, refer to [22].

The ZMMs of the $\mathrm{LL}_{3}$ subband in the IWT domain of an image have semi-fragile characteristics. That is, they meet the following requirements:

i) High sensitivity to malicious modification.

ii) Robustness to incidental modification.

Such characteristics can be evaluated by computing the difference between the ZMMs of the original image and that of the image suffered from various attacks. Root-mean-square-error (RMSE) may be used to measure the difference of ZMMs. The RMSE of ZMMs is defined as below.

$$
\Delta=\sqrt{\frac{1}{N} \sum_{i=1}^{N}\left(M_{i}-M_{i}^{\prime}\right)^{2}}
$$

where $\Delta$ denotes RMSE of ZMMs, $M_{i}$ and $M_{i}^{\prime}$ denote the ZMMs of $\mathrm{LL}_{3}$ subband in the IWT domain of an image before and after attack, respectively. $N$ is the numbers of the ZMMs of the $\mathrm{LL}_{3}$ subband in the IWT domain of an image. Because the Zernike moments of order 12 have a moderate computational complexity and are enough to represent image features, therefore we compute Zernike moments of order 12 , total of 49 moments here. We demonstrate such characteristics by making an experiment on two hundred images of $512 \times 512 \times 8$ bits. Results on statistical quantities (mean and standard deviation) of RMSE of ZMMs for these images are shown in Table 1.

In Table 1, JPEG90 refers to JPEG compression with a quality factor of 90. Noise1, Noise2, Noise3 refer to zero-mean additive Gaussian noise with variance 0.0001 , $0.0005,0.0009$, respectively. In our experiment, cut refers to replace a region with all white pixels. Replace refers to replace a region by another region from the same image or from another image. From Table 1, we can observe that mean and standard deviation of RMSE of ZMMs of JPEG40 and Noise3 are (128.59, 32.37) and (145.19, 55.08), respectively. The mean and standard deviation of RMSE of ZMMs of cut $(16 \times 16)$ and 
replace $(16 \times 16)$ are $(5067.88,3419.79)$ and $(470.83,248.12)$, respectively. The mean and standard deviation of RMSE of ZMMs of cut $(32 \times 32)$ and replace $(32 \times 32)$ are $(5366.18,3233.03)$ and $(807.74,437.63)$, respectively. That is if an image is suffered from incidental modifications, such as JPEG and noise, the differences of ZMMs are far smaller than those of malicious attack, such as cut and replace. It indicates that the semifragile characteristic of ZMMs of the $\mathrm{LL}_{3}$ subband in the IWT domain of an image. Using this feature, we can distinguish incidental distortion from severe distortion according to a pre-selected threshold.

Table 1. Statistical quantities of RMSE of ZMMs of images before and after attack

\begin{tabular}{|c|c|c|}
\hline Attack & Mean & Standard Deviation \\
\hline JPEG90 & 54.05 & 35.35 \\
\hline JPEG70 & 74.78 & 45.22 \\
\hline JPEG50 & 100.01 & 28.92 \\
\hline JPEG40 & 128.59 & 32.37 \\
\hline Noise 1 & 105.37 & 50.04 \\
\hline Noise 2 & 120.33 & 47.44 \\
\hline Noise 3 & 145.19 & 55.08 \\
\hline Cut $(16 \times 16)$ & 5067.88 & 3419.79 \\
\hline Replace $(16 \times 16)$ & 470.83 & 248.12 \\
\hline $\operatorname{Cut}(32 \times 32)$ & 5366.18 & 3233.03 \\
\hline Replace $(32 \times 32)$ & 807.74 & 437.63 \\
\hline
\end{tabular}

\section{The Proposed Scheme}

\subsection{Outline of the Scheme}

Fig. 1 and Fig. 2 are block diagrams of the watermark embedding, tamper detection and recovery procedure of the proposed scheme, respectively.

When embedding, we first pre-process the original image by histogram modification to prevent overflow/underflow. Then, we perform 3-level IWT on the pre-processed image. In the meantime, we compute Zernike moments of $\mathrm{LL}_{3}$ subband of the preprocessed image. We embed watermark in $\mathrm{HH}_{3}$ subband and the recovery information (bookkeeping date, original $1^{\text {st }}$ bit plane of $\mathrm{HH}_{3}$ subband etc.) in $\mathrm{HH}_{1}, \mathrm{LH}_{1}$ and $\mathrm{HL}_{1}$ subband. Finally, inverse IWT is applied and the marked image is obtained.

In tamper detection and recovery stage, we first perform 3-level IWT on the suspected image. Then we compute Zernike moments of $\mathrm{LL}_{3}$ subband of the 


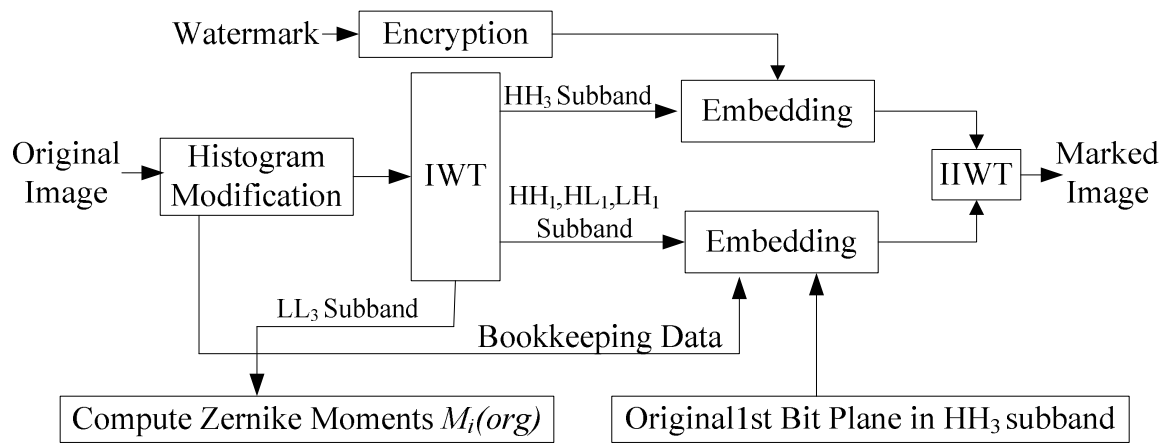

Fig. 1. Watermark embedding

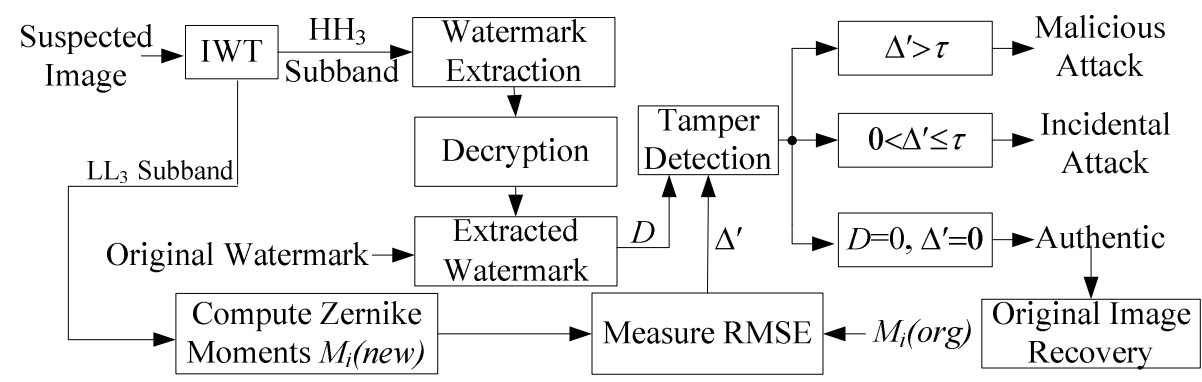

Fig. 2. Tamper detection and recovery

suspected image. Watermark is extracted from $\mathrm{HH}_{3}$ subband. Finally, according to the difference image of watermark $D$ and RMSE of Zernike moments $\Delta^{\prime}$, we judge the image is authentic, incidental attacked or malicious attacked. If the image is authentic, we further recover the original image.

More details will be described in Section 3.2 and Section 3.3.

\subsection{Reversible Watermark Embedding}

The bit-plane of IWT coefficients in $\mathrm{HH}_{1}, \mathrm{LH}_{1}$ and $\mathrm{HL}_{1}$ subbands has the following property: The higher the bit-plane, the larger the bias between 0's and 1's. By compressing bits in this bit-plane, it leaves room to hide data [10].

We use this property to implement reversible watermark embedding. Although the higher bit-plane may embed much more data, it will cause higher distortion. Considering lower embedding capacity, we choose the $4^{\text {th }}$ bit-plane of the IWT coefficients in the $\mathrm{HH}_{1}, \mathrm{LH}_{1}$ and $\mathrm{HL}_{1}$ subbands, which ensures sufficient space and better marked image quality, to hide data. We use a binary image, $W$, as the watermark for tamper detection. We encrypt it in consideration of security and denote the result as $W^{*}$. The embedding procedures are described as follows. 
i) Pre-process the original image by carrying out histogram modification to prevent overflow/underflow [10]. In order to restore the original image losslessly later, the bookkeeping information of histogram modification should be hidden as overhead. We denote it as $B$.

ii) Compute $49 \mathrm{ZMMs}$ of $\mathrm{LL}_{3}$ subband in IWT domain of the pre-processed image. Denote them $M_{i}($ org $), 1 \leq i \leq 49$. These 49 ZMMs needs to be transmitted to the receiver as the side information for distinguishing malicious attack from non-malicious attack.

iii) Denote the $1^{\text {st }}$ bit-plane of the IWT coefficients in $\mathrm{HH}_{3}$ subband as $L$. We replace $L$ with $W^{*}$ to embed mark data.

iv) Losslessly compress the original $4^{\text {th }}$ bit-plane of the IWT coefficients in $\mathrm{HH}_{1}, \mathrm{LH}_{1}$ and $\mathrm{HL}_{1}$ subbands using arithmetic coding. Let $C$ denote the compressed data.

v) Combine $B, C$ and $L$ into a bit stream $S$. Substitute $S$ for the original $4^{\text {th }}$ bitplane of the IWT coefficients in $\mathrm{HH}_{1}, \mathrm{LH}_{1}$ and $\mathrm{HL}_{1}$ subbands.

vi) Perform the inverse IWT to generate a watermarked image.

In this way, we implement reversible watermark embedding.

\subsection{Tamper Detection and Recovery}

To a suspected image, the authentication is performed via the following steps.

i) Compute $49 \mathrm{ZMMs}$ of $\mathrm{LL}_{3}$ subband in IWT domain of a suspected image. Denote them $M_{i}^{\prime}($ new $), 1 \leq i \leq 49$.

ii) Extract the hidden watermark in the $1^{\text {st }}$ bit-plane of the IWT coefficients in $\mathrm{HH}_{3}$ subband. Via decryption, we denote the result as $W^{\prime}$.

iii) Compute the difference image $D$ and $\Delta^{\prime}$ of ZMMs of $\mathrm{LL}_{3}$ subband in IWT domain according to formula (7) and (8)

$$
\begin{gathered}
D=\left|W-W^{\prime}\right| \\
\Delta^{\prime}=\sqrt{\frac{1}{49} \sum_{i=1}^{49}\left(M_{i}(\text { org })-M_{i}^{\prime}(\text { new })\right)^{2}}
\end{gathered}
$$

According to formula (7), if $W$ is equal to $W^{\prime}$, all pixels in the difference image have value 0 . Otherwise, some pixels in the difference image have value 1 which means mark extraction error. Thus white pixel in the difference image $D$ represents the pixel is tampered. Hence, we can locate the tampered area according to $D$.

iv) If $D$ and $\Delta^{\prime}$ are all equal to zero, the image is supposed to be authentic. Extract $B, C$ and $L$ from the $4^{\text {th }}$ bit-plane of $\mathrm{HH}_{1}, \mathrm{LH}_{1}$ and $\mathrm{HL}_{1}$ subbands. Using these information, the original image can be recovered from the marked image losslessly.

v) If $\Delta^{\prime}$ is not equal to zero, let $\tau$ be a threshold which can be determined empirically to distinguish malicious tamper from incidental attack. If $\Delta^{\prime} \leq \tau$, 
we decide that the image suffers from incidental modification. Otherwise, it is malicious attacked. In the latter case, if $D$ is equal to zero, we conclude the image has suffered from forgery attack mentioned in introduction. So this forgery image can not pass authentication thus improving security. If $D$ is not equal to zero, we can further locate the tampered region.

\section{Experimental Results}

We test the effectiveness of our proposed algorithm using the images shown in Fig. 3 . The PSNRs of the watermarked images are shown in Table 2. Specifically, the marked images of Baboon and Peppers are shown in Fig. 4. We can observe no difference between the marked image and the corresponding original image. Fig. 5 shows the results of the fragility to malicious tamper such as cut and replacement. It is obvious that our algorithm can detect malicious modifications and locate the tampered areas accurately. Table 3 is the difference of ZMMs for Baboon and Peppers that suffered from malicious attack and JPEG compression. The cut and replace operations are shown in Fig. 5. It is seen that setting a threshold value (e.g. 200) will distinguish JPEG compression from cut and replace operation. Moreover, it also demonstrates that our scheme can tolerate JPEG lossy compression with a quality factor as low as 40. Since images compressed at a quality factor smaller than 40 may lead to obvious perceptual distortion, robustness to JPEG at a quality factor 40 is enough in most of applications.

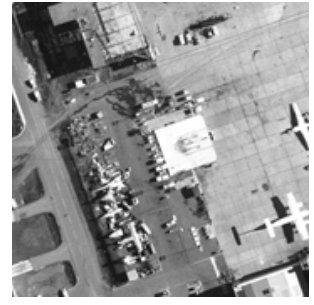

(a) Airfield

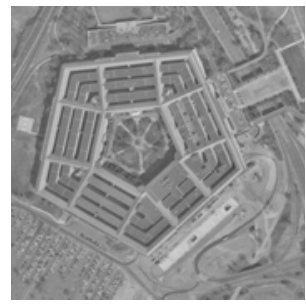

(d) Pentagon

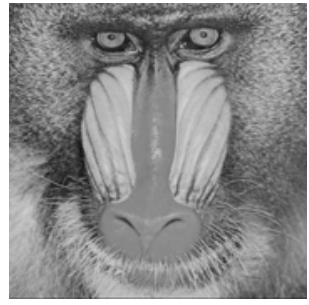

(b) Baboon

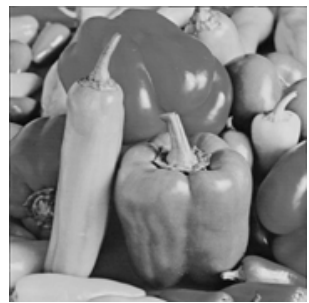

(e) Peppers

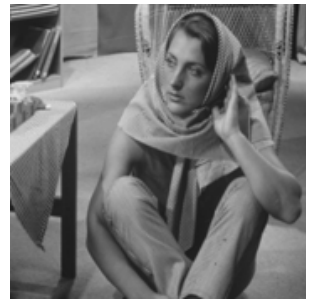

(c) Barb

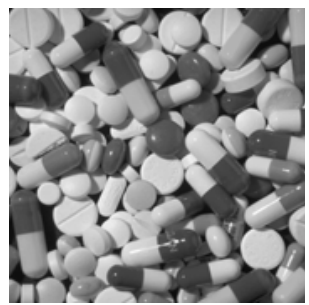

(f) Pills

Fig. 3. Six original images 


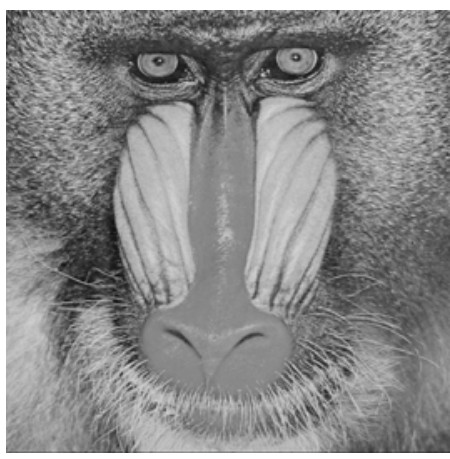

(a)

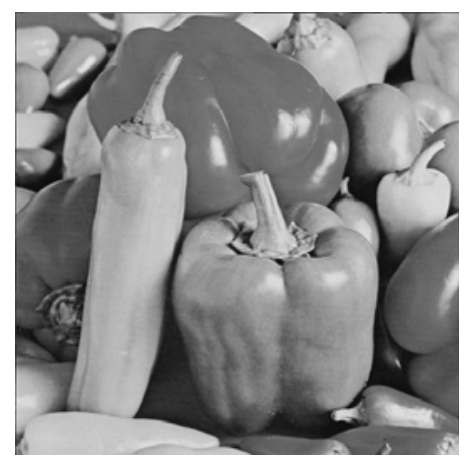

(b)

Fig. 4. Watermarked image (a) Baboon; (b) Peppers

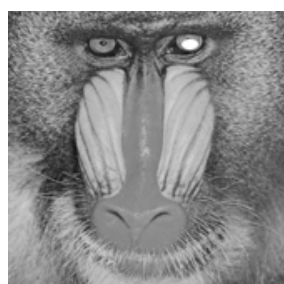

(a)

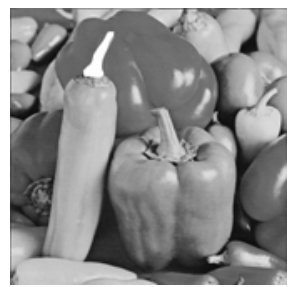

(e)

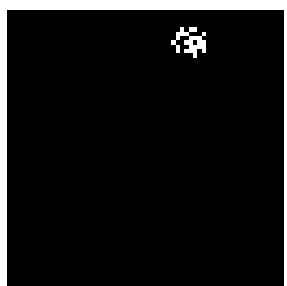

(b)

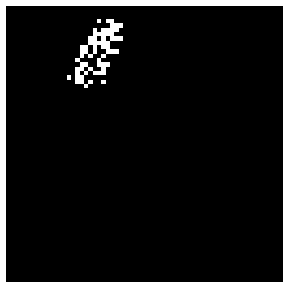

(f)

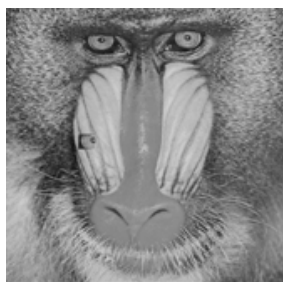

(c)

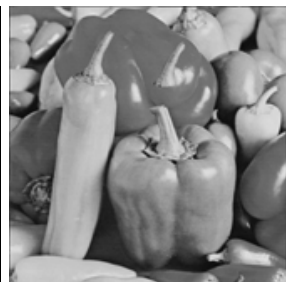

(g)

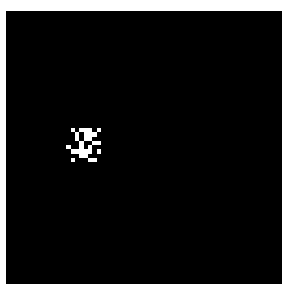

(d)

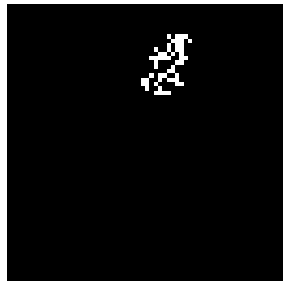

(h)

Fig. 5. Tamper Detection. (a), (c), (e), (g) Tampered images; (b), (d), (f), (h) Difference images of extracted watermarks.

Table 2. PSNR of six marked images

\begin{tabular}{|c|c|c|c|}
\hline Test image & PSNR of marked image $(\mathrm{dB})$ & Test image & PSNR of marked image $(\mathrm{dB})$ \\
\hline Airfield & 35.54 & Pentagon & 36.91 \\
\hline Baboon & 37.88 & Peppers & 39.04 \\
\hline Barb & 36.72 & Pills & 37.44 \\
\hline
\end{tabular}


Table 3. Diferrence of ZMMs of $\mathrm{LL}_{3}$ subband in IWT domain of an image suffered from various attack

\begin{tabular}{|c|c|c|c|c|c|c|}
\hline image attact & JPEG90 & JPEG70 & JPEG50 & JPEG40 & Cut & Replace \\
\hline Baboon & 47.99 & 73.36 & 145.04 & 158.56 & 5257.90 & 523.94 \\
\hline Peppers & 60.66 & 74.65 & 82.50 & 86.95 & 3685.70 & 1352.20 \\
\hline
\end{tabular}

\section{Conclusions}

In this paper, we propose a novel reversible semi-fragile authentication algorithm for images based on Zernike moments and integer wavelet transform. The main contributions are as follows:

i) The proposed algorithm is able to recover an original image from the marked image losslessly after the watermarked image is authenticated.

ii) Using semi-fragile characteristics of Zernike moments, the proposed algorithm is able to distinguish malicious modifications from incidental modifications.

iii) Combining semi-fragile characteristics of ZMMs, the watermark can discern forgery attack and thus improve the watermark security.

iv) The proposed algorithm is capable of detecting tamper accurately while tolerat ing JPEG lossy compression at a low quality factor.

In our future research, we will focus on designing an authentication scheme that can accept incidental geometrical manipulations such as scaling, rotations.

\section{Acknowledgement}

Supported by NSF of China (60325208, 60403045, 90604008), NSF of Guangdong (04205407), Key Project of Science and Technology of Guangzhou, China (2005Z3D0391).

\section{References}

1. M. M. Yeung, F. Mintzer, "An invisible watermarking technique for image verification," Proc. of IEEE Int. Conf. on Image Processing, vol. 2, pp. 680-683, 1997

2. P. W. Wong, N. Memon, "Secret and public key image watermarking schemes for image authentication and ownership verification," IEEE Trans. on Image Processing, 10(10):1593-1601, 2001

3. C. T. Li, F. M. Yang and C. S. Lee, "Oblivious fragile watermarking scheme for image authentication," Proc. of IEEE Int. Conf. on Acoustics, Speech, and Signal Processing, vol. 4, pp. 3445-3448, 2002

4. D. Kundur, D. Hatzinakos, "Towards a telltale watermark techniques for tamperproofing," Proc. of IEEE Int. Conf. on Image Processing, vol. 2, pp. 409-413, 1998 
5. C. Y. Lin, S. F. Chang, "Semi-fragile watermarking for authenticating JPEG visual content," Proc. of SPIE Security and Watermarking of Multimedia Content II, vol. 3971, pp. $140-151,2000$

6. J. Hu, J. Huang, D. Huang and Y. Shi, "Image fragile watermarking based on fusion of multi-resolution tamper detection," IEE Electronics Letters, 38(24):1512-1513, 2002

7. Z. Lu, C. Liu, D. Xu and S. Sun, "Semi-fragile image watermarking method based on index constrained vector quantization," IEE Electronics Letters, 39(1): 35-36, 2003

8. B. Zhu, M. D. Swanson, A. H. Tewfik, "When Seeing isn't Believeing," IEEE Trans. on Signal Processing, 21(2): 40-49, 2004

9. Y. Shi, Z. Ni, D. Zou, C. Liang and G. Xuan, "Lossless data hiding: fundamentals, algorithms and applications," Proc of IEEE Int. Conf. on Circuits and Systems, vol. 2 pp. 33-36, 2004

10. G. Xuan, J. Zhu, J. Chen, Y. Shi, Z. Ni and W. Su, "Distortionless data hiding based on integer wavelet transform," IEE Electronics Letters, 38(25): 1646-1648, 2002

11. M. Goljan, J. Fridrich and R. Du, "Distortion-free data embedding," Proceedings of $4^{\text {th }}$ Information Hiding Workshop, pp. 27-41, 2001

12. J. Tian, "Reversible data embedding using a difference expansion," IEEE Trans. on Circuits and Systems for Video Technology, 13(8):890-896, 2003

13. M. U. Celik, G. Sharma, A. M. Tekalp, E. Saber, "Reversible data hiding," Proc of IEEE Int. Conf. on Image Processing, vol. 2 pp. 157-160, 2002

14. J. M. Barton, "Method and apparatus for embedding authentication information within digital data," U.S. Patent 5,646,997, 1997

15. C. W. Honsinger, P. Jones, M. Rabbani, and J. C. Stoffel, "Lossless recovery of an original image containing embedded data," US Patent: 6,278,791, 2001

16. J. Fridrich, M. Goljan and R. Du, "Invertible authentication," Proc. of SPIE Security and Watermarking of Multimedia Contents III, vol. 4314, pp. 197-208, 2001

17. C. De Vleeschouwer, J. F. Delaigle and B. Macq, "Circular interpretation of bijective transformations in lossless watermarking for media asset management," IEEE Trans. on Multimedia, 5(1):97-105, 2003

18. D. Zou, Y. Shi and Z. Ni, "A semi-fragile lossless digital watermarking scheme based on integer wavelet transform," Proc of IEEE Int. Conf. on Multimedia Signal Processing, pp. 195-198, 2004

19. S. Katzenbeisser, J. Dittmann, "Malicious attacks on media authentication schemes based on invertible watermarks," Proc. of SPIE Security and Watermarking of Multimedia Content VI, vol. 5306 pp. 838-847, 2004

20. I. Daubechies, W. Sweldens, "Factoring wavelet transform into lifting step," Journal of Fourier Analysis, vol. 4 pp. 245-267, 1998

21. R. Calderbank, I. Daubechies, W. Sweldens, B.L. Yeo, "Wavelet transforms that map integers to integers," Journal of Applied and Computational Harmonic Analysis, vol. 5 pp. 332-369, 1998

22. A. Khotanzad, Y. H. Hong, "Invariant image recognition by Zernike moments," IEEE Trans. on Pattern Analysis and Machine Intelligence, 12(5):489-497, 1990 